\title{
Transient nanobubbles in short-time electrolysis
}

\author{
Vitaly B. Svetovoy, ${ }^{1,2, *}$ Remco G. P. Sanders, ${ }^{1}$ and Miko C. Elwenspoek ${ }^{1,3}$ \\ ${ }^{1} \mathrm{MESA}^{+}$Institute for Nanotechnology, University of Twente, PO 217, 7500 AE Enschede, The Netherlands \\ ${ }^{2}$ Institute of Physics and Technology, Yaroslavl Branch, \\ Russian Academy of Sciences, 150007, Yaroslavl, Russia \\ ${ }^{3}$ FRIAS, University of Freiburg, 79104 Freiburg, Germany
}

(Dated: October 15, 2018)

\begin{abstract}
Water electrolysis in a microsystem is observed and analyzed on a short-time scale $\sim 10 \mu$ s. Very unusual properties of the process are stressed. An extremely high current density is observed because the process is not limited by the diffusion of electroactive species. The high current is accompanied by a high relative supersaturation $S>1000$ that results in homogeneous nucleation of bubbles. On the short-time scale only nanobubbles can be formed. These nanobubbles densely cover the electrodes and aggregate at a later time to microbubbles. The effect is significantly intensified with a small increase of temperature. Application of alternating polarity voltage pulses produces bubbles containing a mixture of hydrogen and oxygen. Spontaneous reaction between gases is observed for stoichiometric bubbles with the size smallaer than $150 \mathrm{~nm}$. Such bubbles disintegrate violently affecting the surface of electrodes.

PACS numbers: 47.55.D-, 64.60.qj, 82.33.Vx
\end{abstract}

\section{INTRODUCTION}

More than 200 years gone since people observed water electrolysis for the first time (see [1, 2] and references therein). Since then the process is widely used in different applications including hydrogen production $[3,4]$. Nowadays electrolysis of water find also applications in different kind of microdevices such as actuators [5 8], pumps [9, 10], and others [11, 12]. Fast performance of microsystems requires processing on a short-time scale. Surprisingly enough, most of the researches on water electrolysis dealt with macro or microscopic systems on a time scale longer than milliseconds 9, 13]. A few papers, where shorter (microseconds) voltage pulses were considered, discussed increase of the overall efficiency 14 or optimization of the performance of electrolysis [15], but did not observe the process on the microsecond time scale.

In normal long-time electrolysis (long-time means here the time scale $\tau>1 \mathrm{~ms}$ ) gas bubbles with the diameters in the range $10-1000 \mu \mathrm{m}$ are observed [13, 16]. The maximal current density that can be reached in this case is around $1 \mathrm{~A} \cdot \mathrm{cm}^{-2}$ [17 and the maximal relative supersaturation $\sim 100[18$. These values cannot be increased further because bubbles cover all the surface of the electrode making it inactive [17, 19, 20].

In the short-time electrolysis, say $\tau=1-100 \mu \mathrm{s}$, one could expect unusual properties of the process. It becomes obvious if we consider gas diffusion into the liquid above the electrode. For the time $\tau$ a significant supersaturation is possible at the distance from the electrode $l \sim \sqrt{D \tau}$, where $D$ is the diffusion coefficient of the formed gas in the surrounding liquid. Taking as typ-

*Corresponding author: v.svetovoy@utwente.nl ical values $D \sim 10^{-9} \mathrm{~m}^{2} / \mathrm{s}$ and $\tau \sim 10 \mu$ s one finds $l \sim 100 \mathrm{~nm}$. It means that on this timescale only nanobubbles can be formed.This is indeed the case as we demonstrated recently [21]. Nanobubbles of 200-300 nm in diameter were observed stroboscopically above microelectrodes. These nanobubbles were formed for the first $20-50 \mu$ s then aggregated to microbubbles after $100-300 \mu \mathrm{s}$, but the following dynamics was very slow.

A number of observations indicate that smaller nanobubbles can exist at shorter times $\tau \lesssim 10 \mu$ s. For example, it was demonstrated that the reaction between hydrogen and oxygen can be ignited spontaneously in nanobubbles with the size smaller than $150 \mathrm{~nm}$ [21]. Different manifestations of this reaction show that the reaction persists for nanobubbles as small as $50 \mathrm{~nm}$ or even smaller.

In this paper we describe formation of nanobubble on the short-time scale $\tau=1-100 \mu \mathrm{s}$. It is demonstrated that very high current densities and supersaturations can exist accompanied by the homogeneous nucleation of nanobubbles. The bubbles containing hydrogen or oxygen grow and aggregate forming micro-sized bubbles on the timescale $\tau \gtrsim 100 \mu \mathrm{s}$. The nanobubbles containing mixture of the gases disappear as the result of the reaction between gases. These are transient nanobubbles, which exist only a short time and difficult for direct observation. In contrast with the first report [21] we provide here detailed description of the transient nanobubbles and pay less attention to the chemical reaction inside of these bubbles.

The nanobubbles discussed in this paper differ from the surface nanobubble, which attracted recently a considerable attention. The long-lived surface nanobubbles were observed with different methods 22 24]. Observation with atomic force microscopes (AFM) became especially popular (see [25] and references therein) due to its simple realization. These nanobubbles are widely dis- 
cussed in the literature because of their mysterious stability. High Laplace pressure drives diffusion of gas out of the bubble resulting in a dissolution time of $\sim 10 \mu \mathrm{s}$ but not days as observed (see [26] for a review). Precise reason for this stability is not clear yet but in a promising model [27] of "dynamical equilibrium" the gas flux going out of the bubble through the spherical cap is balanced with a gaseous influx at the contact line.

A number of methods to generate the surface nanobubbles are in use including solvent exchange, temperature change for liquid or substrate, and liquid pressurizing [28]. The electrochemical formation of the surface nanobubbles were observed in water electrolysis for hydrogen [29] and for both hydrogen and oxygen [30] on highly orientated pyrolytic graphite. The electrochemical process is considered as a flexible way to control the bubble production (density and size) [31]. In contrast with the electrochemical process considered in this paper the electrochemical production of the surface nanobubbles had much smaller current density $\left(\sim 10^{-4} \mathrm{~A} / \mathrm{cm}^{2}\right)$ and much longer time scale $(\sim 10 \mathrm{~s})$.

This paper is organized as follows. In Sec. II we describe the system for observation of the short-time electrolysis, application of the voltage pulses, and the current response. In Sec. III the main results obtained with the stroboscopic observations are described. Electrolysis with alternating sign pulses is described in Sec. IV where we present the results obtained with a stroboscop and vibrometer. We explain also modification of the electrode surface due to the process. In Sec. V the evolution of the transient nanobubbles is summarized and we discuss their possible applications. Our conclusions are collected in the last section.

\section{SHORT-TIME ELECTROLYSIS}

To analyze electrolysis on a short-time scale in a microscopic system special microchips were prepared. One of the chips is shown in Fig. 1, For fabrication we used wafers made of borofloat glass BF-33. First, Ti sublayer was deposited on glass $(10 \mathrm{~nm})$ for better adhesion, then the $100 \mathrm{~nm}$ thick metallic layer was sputtered. We used the wafers with different metals on top such as $\mathrm{Pt}, \mathrm{Pd}$, $\mathrm{Au}$, or $\mathrm{W}$. The metals were patterned and covered lithographically with the insulating resist SU8 where it was necessary. Different thicknesses of SU8 layer of 3 and $90 \mu \mathrm{m}$ were in use. The fabricated wafers were diced on separate chips of size $12 \times 12 \mathrm{~mm}^{2}$. Each chip contained 16 pairs of the microelectrodes of different shapes and sizes and the contact pads to address a specific pair electrically. The patterned SU8 layer formed walls of open channels for a liquid. A chip filled in with the electrolyte was covered by a thing $(30 \mu \mathrm{m})$ glass plate for observation of the electrolysis.

As the solution for electrolysis we used $15 \mathrm{~g}$ of $\mathrm{Na}_{2} \mathrm{SO}_{4}$ dissolved in $100 \mathrm{ml}$ of deionized water. Also we have used the solutions of $\mathrm{NaCl}$ and $\mathrm{KI}$ in water in similar

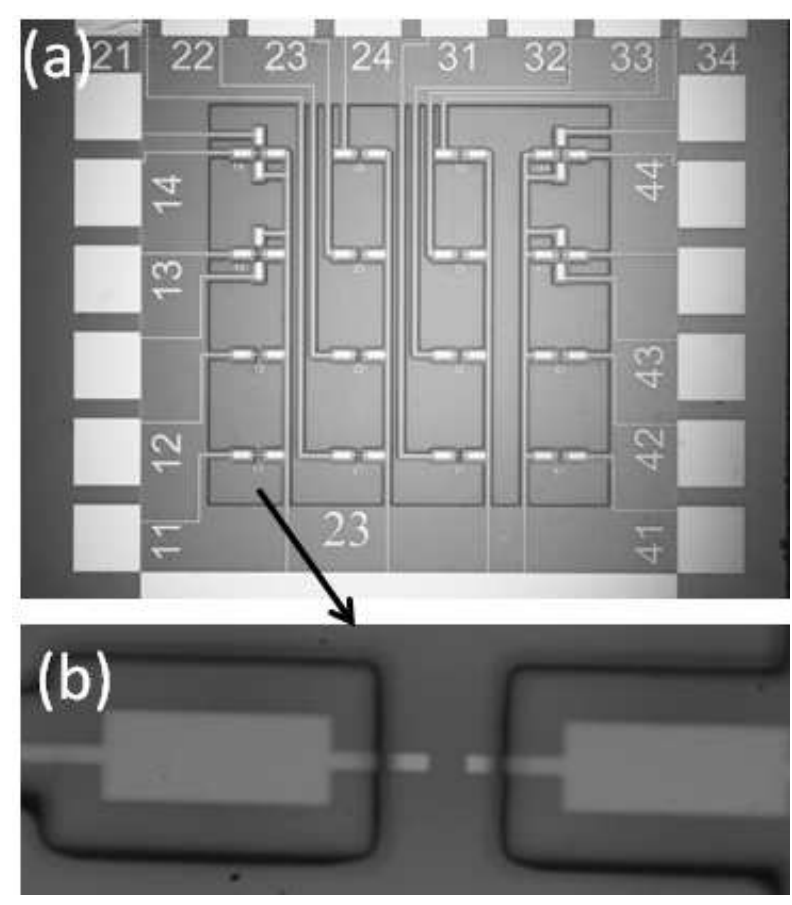

FIG. 1: (a) Microchip used for investigation of electrolysis on short-time scales. (b) Pair of microelectrodes that can be addressed separately.

concentrations.

The process was observed with a homemade stroboscopic system [32]. In this system the light source and the electrical pulses applied to the electrodes were controlled with the time resolution $0.1 \mu \mathrm{s}$. As a light source two powerful green LEDs LUXEON ${ }^{\circledR}$ III were used. With this system we have been able to observe the electrodes in an optical microscope with the illumination time longer than $5 \mu \mathrm{s}$.

\section{A. Voltage}

For a chosen pair of the electrodes one was always grounded and the other one (working electrode) could be at a negative or positive potential. Hydrogen or oxygen bubbles were produced above the working electrode. We could apply voltage as a pulse of a given length from 1 to $10^{5} \mu \mathrm{s}$. The pulse could be repeated with a frequency $f$. At a prescribed moment of time a short flash of light $\geq 5 \mu$ s was on and an optical microscope image of the electrodes was made during the flash. The applied voltage and the resulting current through the electrodes were recorded. Alternatively keeping one electrode grounded we applied voltage pulses of alternating polarity to the working electrode repeated with some frequency. In this case bubbles containing both gases $\mathrm{H}_{2}$ and $\mathrm{O}_{2}$ could be formed [21].

The voltage needed to start the electrolysis was always larger than that for macrosystems, $U_{0}=2.5-4 \mathrm{~V}$. The high threshold voltage was already observed in mi- 
crosystems [7, 9, 10], but the reason was not explained. It has to be stressed that in the microsystems a planar geometry is used when both electrodes are in the same plane. In this case the ohmic losses (resistance between the electrodes) are considerable even for well conducting electrolytes. This is in contrast with the macrosystems, where these losses are usually negligible. For one pair of electrodes we measured the resistance to be $R=2.3 \mathrm{k} \Omega$ but it varies to some degree from pair to pair due to different geometry. The working current in our system $I \sim 1 \mathrm{~mA}$ was quite large. Then the ohmic polarization is estimated as a few volts $(2-3 \mathrm{~V})$.

Conventionally we defined the threshold for the electrolysis applying different voltages for $5 \mathrm{~ms}$ and observing the gas formation. At $U=U_{0}$ the first gas was visible in the system. For example, for the same pair of electrodes, for which the resistance was measured, we found $U_{0}=3.2 \mathrm{~V}$. In some cases $U_{0}$ was as large as $6 \mathrm{~V}$ or so, but we have found out that this was due to the potential drop on the electrode surface. Treatment of such chips in $\mathrm{O}_{2}$-plasma significantly reduced $U_{0}$. Probably a film (hydrocarbons) was formed on top of the electrodes during the fabrication process. It should be stressed that the absolute values of the potential has to be taken with care because we deal with the two-electrode system and there is uncertainty in the potential of the working electrode in contrast with the three-electrode system.

\section{B. Current}

The current response to square voltage pulses applied to the working electrode is shown Fig. 2, The response is in accordance with the expectations. One can separate two components in the current. The first one is the faradaic current $I_{F}$ that does not depend on time $t$ for square voltage pulses. The second component is responsible for the charging-discharging of the double layer on the electrode surface and is not related to the electrochemical reaction. It behaves as $I_{1} e^{-t / \tau_{c}}$, where $I_{1}$ is a constant and $\tau_{c}$ is the relaxation time related to the capacitance of the double layer. As the result we fit the current response on the square voltage pulse by a function of three parameters:

$$
I(t)=I_{F}+I_{1} e^{-t / \tau_{c}} .
$$

It has to be stressed that typically in macro and microsystems considered on the long-time scale the faradaic current $I_{F}$ depends on the time because it is diffusion limited. For parallel electrodes the current is proportional to $\sqrt{\tau_{D} / t}$ in accordance with the Cottrell equation [33], where $\tau_{D}$ is the diffusion time for the electroactive species in the solution. In our case the diffusion time is too long to play any role. Roughly it can be estimated as $\tau_{D} \sim L^{2} / D_{a} \sim 100 \mathrm{~ms}$, where $L \sim 10 \mu \mathrm{m}$ is a typical size of our electrodes and $D_{a} \sim 10^{-9} \mathrm{~m}^{2} / \mathrm{s}$ is a typical diffusion coefficient of the electroactive species in water.

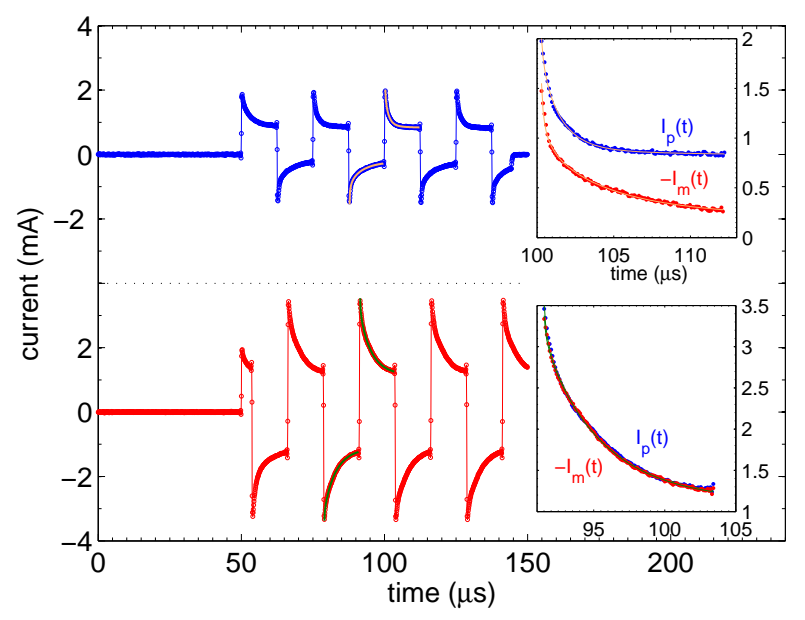

FIG. 2: (top) Current response to the positive square voltage pulses of the amplitude $U=5.2 \mathrm{~V}$ and frequency $f=40 \mathrm{kHz}$. (bottom) Response to the voltage pulses of alternating polarity $U=6 \mathrm{~V}, f=40 \mathrm{kHz}$. The insets show the currents from the main graph for half of one period and the fits of the currents with Eq. 1

We used Eq. (1) to fit the current response as shown in Fig. 2, The quality of the fit can be seen in the insets. The faradaic current can be well approximated by a constant in the investigated time domain $t<100 \mu \mathrm{s}$. For alternating pulses the positive $I_{p}(t)$ and negative $I_{m}(t)$ halves of the pulse are symmetric as shown in the bottom inset. For single polarity pulses the relaxation time $\tau_{c}$ is larger for the negative half of the pulse (4.9 against $1.3 \mu \mathrm{s})$ reflecting dependence of the double layer capacitance on the potential [33]. For alternating polarity pulses in Fig. 2 we have found from the fit $\tau_{c}=4.0 \mu \mathrm{s}$. With the frequency increase the relaxation time decreases slowly indicating that not all adsorption states can be filled for a short time. It has to be noted that the Faraday component of the current persists up to the shortest pulses of $1 \mu \mathrm{s}(f=500 \mathrm{kHz})$, which we investigated. Presence of this component signals formation of gases on this shortest time scale that was directly observed with the stroboscope.

The faradaic current reaches rather large values. For the case shown in Fig. 2 it is $I_{F}=0.80 \pm 0.07 \mathrm{~mA}$ for the single polarity pulses and $I_{F}=1.08 \pm 0.03 \mathrm{~mA}$ for the alternating polarity voltage pulses. Keeping in mind that the open area of the electrode was around $1260 \mu \mathrm{m}^{2}$ one finds that the current density was $j_{F} \sim 80 \mathrm{~A} / \mathrm{cm}^{2}$. This is the average value of the current density but locally it can be 5 times larger (see Sec. IIIA). This value is much larger than the maximal value $\sim 1 \mathrm{~A} / \mathrm{cm}^{2}[17$ ] that can be reached in the long-time electrolysis.

The most important conclusions that can be drawn from the current-voltage behavior of the microsystem on the short-time scale are the following. (i) The electrolysis persists normally up to the shortest investigated time $1 \mu \mathrm{s}$. (ii) The current density is at least two orders of magnitude larger than was previously observed in the 
macrosystems.

\section{STROBOSCOPIC OBSERVATIONS}

The stroboscopic technique gives images that were made for different runs. To make conclusions from these images the system has to behave reproducibly from run to run. For our system this condition was fulfilled. The images made at the same moment for different runs were hardly distinguishable on the short-time scale and varied slightly on the long-time scale $>1 \mathrm{~ms}$. In the latter case the positions and sizes of separate bubbles were slightly different but qualitatively the pictures were the same. For good visibility we used typically a $10 \mu$ s long flash of green light that was delayed for different times from the beginning of the voltage pulse. The camera collected the light from the microscope during the flash. The estimated time uncertainty in $5 \mu \mathrm{s}$ is reasonably small although comparable with the time scale. The gas produced by series of pulses in most cases disappeared diffusively in less than $1 \mathrm{~s}$. Sometimes when the amount of produced gas was large a stable (pinned) bubble formed. In this case we have to refill the chip for further use. Due to evaporation we have been able to operate with a filled chip during 15 min.

Figure 3 shows the electrodes in different moments of time. A positive or negative voltage pulse of $50 \mu \mathrm{s}$ long was applied to the electrodes. The left image shows both electrodes $20 \mu$ s after beginning of the positive voltage pulse. Oxygen and hydrogen are formed above the working electrode and grounded electrode, respectively. All the other images show the working electrode with hydrogen in different moments during the negative voltage pulse.

Indeed, the periphery of the electrode is a preferable place for bubble nucleation, but one can see some haze in the inner part of the electrode. With our microscope we can reliably resolve bubbles with a diameter of $500 \mathrm{~nm}$. The bubbles forming the haze are smaller. We are able to see them because they strongly scatter the light. The diameter of these scattering centers is estimated as $d>$ $\lambda / \pi \approx 170 \mathrm{~nm}[34]$ where $\lambda=520 \mathrm{~nm}$ is the wavelength of the used LED. These scattering centers fill densely the inner part of the electrode and grow with time.

\section{A. Supersaturation}

Let us make simple estimates. For the time $\tau=20 \mu \mathrm{s}$ the gas molecules can diffuse in the vertical direction off the electrode on the distance

$$
l_{H_{2}} \sim \sqrt{D_{H_{2}} \tau} \approx 300 \mathrm{~nm}, \quad l_{O_{2}} \approx 200 \mathrm{~nm},
$$

where $D_{H_{2}}=4.5 \times 10^{-9} \mathrm{~m}^{2} / \mathrm{s}$ and $D_{O_{2}}=2.0 \times 10^{-9} \mathrm{~m}^{2} / \mathrm{s}$ are the diffusion coefficients of hydrogen and oxygen in water. These values of the diffusion length have to be

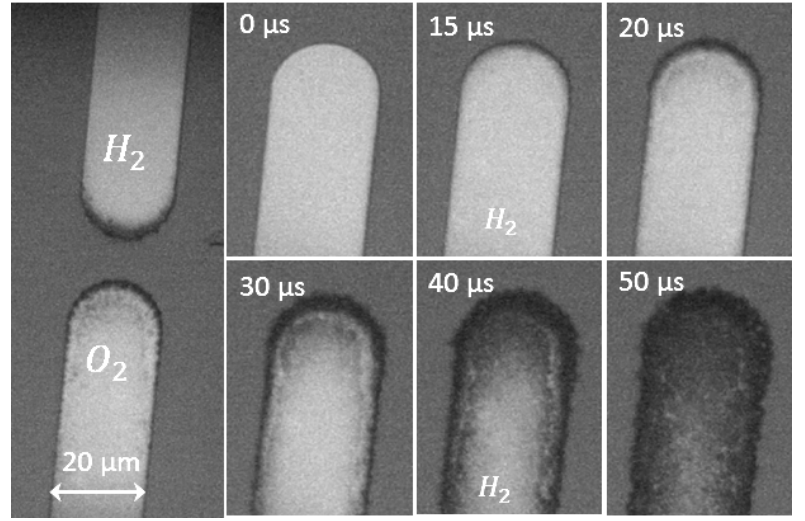

FIG. 3: (left panel) Positive potential is applied to the working electrode (bottom). The photo was made $20 \mu$ s after switching on the voltage. Six images on the right show the working electrode in different moments of time when negative voltage is applied to it. The indicated time is taken in the middle of the flash.

considered as the upper limits on the bubbles that can be formed during the time $\tau$. Therefore, it is natural that we can see only nanobubbles during the first $20 \mu \mathrm{s}$ of electrolysis.

The relative supersaturation for $\mathrm{H}_{2}$ or $\mathrm{O}_{2}$ molecules can be found as follows. The concentration of $i$-th gas in the diffusion layer is $n_{i}=\mathcal{N}_{i} / l_{i} A$, where $A$ is the area of the working electrode and $\mathcal{N}_{i}$ is the number of molecules produced by the electrolysis. This number is related to the Faraday current as $\mathcal{N}_{H_{2}}=I_{F} \tau / 2|e|\left(\right.$ for $\left.\mathrm{H}_{2}\right)$, where $e$ is the electron charge. The relative supersaturation is the ratio of the gas concentration to the saturated concentration $n_{i}^{(s)}$, which at normal conditions is $4.7 \times 10^{17} \mathrm{~cm}^{-3}$ or $7.7 \times 10^{17} \mathrm{~cm}^{-3}$ for hydrogen or oxygen, respectively. Then we find for the supersaturation

$$
S_{H_{2}}=\frac{j_{F}}{2|e| n_{H_{2}}^{(s)}} l_{H_{2}}, \quad S_{O_{2}}=\frac{j_{F}}{4|e| n_{O_{2}}^{(s)}} l_{O_{2}}
$$

where $j_{F}=I_{F} / A$ is the current density.

The faradaic current recorded together with the images in Fig. 3 was $I_{F} \approx 1.5 \mathrm{~mA}$. However, it is not straightforward to estimate the current density because it is highly nonhomogeneous for the planar electrodes. We already analyzed the current distribution over the electrode (see 21] Supplemental) extracting the information from the observed "wear" of the electrodes. It was found that the current density over the electrode surface can be presented as $j_{F}(x, y)=j_{m} f(x, y)$, where $x$ and $y$ are the coordinates of a point on the electrode and $j_{m}$ is the maximal current density. The function $f(x, y)$ has its average value over the electrode $\overline{f(x, y)} \approx 0.2$. The area of the electrode was $A \approx 1260 \mu \mathrm{m}^{2}$ and we find $j_{m} \approx 600 \mathrm{~A} / \mathrm{cm}^{2}$. This is an extremely high value in comparison with usual electrolysis and it is realized in the area of active bubble formation as can be seen in Fig. 3. The supersaturation in this area for the time 
$\tau=20 \mu \mathrm{s}$ is estimated as

$$
S_{H_{2}}^{m} \approx 2700, \quad S_{O_{2}}^{m} \approx 1200
$$

These are incredibly large values.

\section{B. Homogeneous bubbling}

Observation of the haze far from the electrode periphery is a signal of homogeneous nucleation of small bubbles. Our estimates of the supersaturation (4) also support this idea. In the classical theory of homogeneous nucleation [35, 36] the probability to create a bubble of the critical size $r_{c}$ is given by the exponent

$$
w \sim \exp \left\{-\frac{4 \pi r_{c}^{2} \gamma}{3 k_{B} T}\right\}, \quad r_{c}=\frac{2 \gamma}{\Delta P}
$$

where $\gamma$ is the surface tension of the liquid and $\Delta P$ is the pressure difference between gas and liquid. The pressure in gas $P_{g}$ must support the high supersaturation in liquid. It is defined as $P_{g}=S P_{s}$, where $P_{s}$ is the saturated pressure for a gas at normal conditions. For both $\mathrm{H}_{2}$ and $\mathrm{O}_{2}$ gases $P_{s} \approx 1$ atm and we find $\Delta P_{i} \approx P_{a}\left(S_{i}-1\right)$ for the $i$-th gas.

For small supersaturation $S-1 \sim 1$ the critical size $r_{c} \sim 1 \mu \mathrm{m}$ is large and the exponent in (5) is incredibly small. For high supersaturation $S \sim 1000$ the critical bubble $r_{c} \sim 1 \mathrm{~nm}$ is small and the probability to create a critical bubble, which is able to grow, becomes appreciable. It is hardly reasonable to make more detailed estimates. This is because a number of important corrections have to be included in the classical nucleation theory [35, 37, 38]. It has to be noted that according to (3) $S_{i}$ increases with time as $\sqrt{\tau}$. Therefore, starting from some moment after beginning of the electrolysis homogeneous bubble formation is expected. With the temperature increase as one can see from (5) the homogeneous bubbling must start earlier and due to the exponential dependence this tendency must be well pronounced. This prediction is very easy to check experimentally doing water electrolysis at elevated temperatures.

For this experiment we heated the chip by a flat resistor and controlled the temperature by a thermocouple. A $20 \mu$ s long negative voltage pulse was applied to the working electrode. Images shown in Fig. 4 were taken at the moments $t=17 \mu \mathrm{s}$ (top row) just before the pulse end and at $t=27 \mu$ s (bottom row) $7 \mu$ s after the end of the pulse. Some chemical remnants are clearly visible on the electrode surface. They appeared due to imperfections in the fabrication process and visualize the area with the reduced ability for gas production. The temperature increase resulted in the increase of the Faraday current from $I_{F} \approx 1.3 \mathrm{~mA}$ at $T=23^{\circ} \mathrm{C}$ up to $I_{F} \approx 2.5 \mathrm{~mA}$ at $T=53^{\circ} \mathrm{C}$. This increase is expected due to the increase of the ions diffusion coefficients. However, the increase of the bubble visibility and speed up of the dynamics are much more prominent.

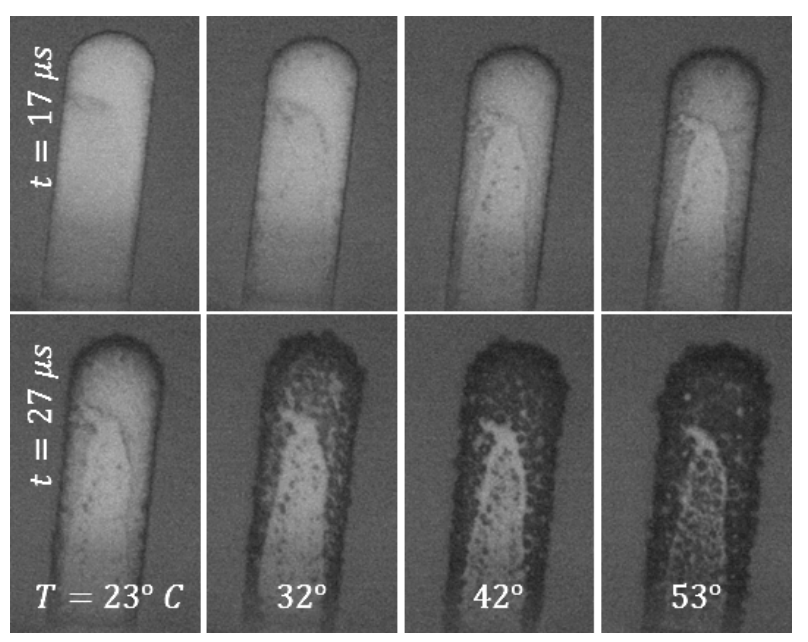

FIG. 4: Images of the electrode at $t=17 \mu \mathrm{s}$ (top row) and at $t=27 \mu$ s (bottom row) taken at different temperatures. A negative voltage pulse of $20 \mu$ s long was the applied to the electrode. Hydrogen nano and microbubbles are visible.

Increase of the solution conductivity (or equivalently current) with temperature was checked independently by measuring the conductivity of the bulk solution with Mettler Toledo SevenMulti conductivity meter. We have found that the conductivity can be well described by the linear behavior $\sigma(T)=\sigma_{0}\left[1+\alpha\left(T-T_{0}\right)\right]$, where $\sigma_{0}$ is the conductivity at temperature $T_{0}$. The thermal coefficient was found to be $\alpha=0.024 \mathrm{~K}^{-1}$. This value is in a good agreement with the value $0.028 \mathrm{~K}^{-1}$ found from the current increase on the chip and with the values reported in different literature sources.

Comparing the images in the top row of Fig. 4 one can see that much more nanobubbles becomes visible with the temperature increase. On the other hand, the gas volume at $T=53^{\circ} \mathrm{C}$ must be approximately twice larger than at $T=23^{\circ} \mathrm{C}$ as follows from the current increase. It has the following explanation. At higher temperature the nucleation of critical bubbles happened earlier and to the moment $t=17 \mu$ s much more bubbles had enough time to grow to a visible size. At $T=23^{\circ} \mathrm{C}$ most of the bubbles are small enough to be invisible.

It becomes even more clear when one compares the top and bottom rows. For example, the bottom image at $23^{\circ} \mathrm{C}$ contains much more visible gas than the top image. However, when the pulse is over there is no gas production anymore and one would expect the equal or the opposite relation between the gas volumes. There is a very simple and natural explanation for this phenomenon. The voltage pulse produces a certain amount of gas. This gas can exist in three forms: molecules dissolved in the liquid, nanobubbles larger than the critical size but smaller than needed to be visible with the stroboscope, and visible nano or microbubbles. If homogeneous nucleation of bubbles happens, then at a certain moment a significant volume of gas is collected in the invisible nanobubbles. These nanobubbles grow with time and become visible with a delay producing the phenomenon 


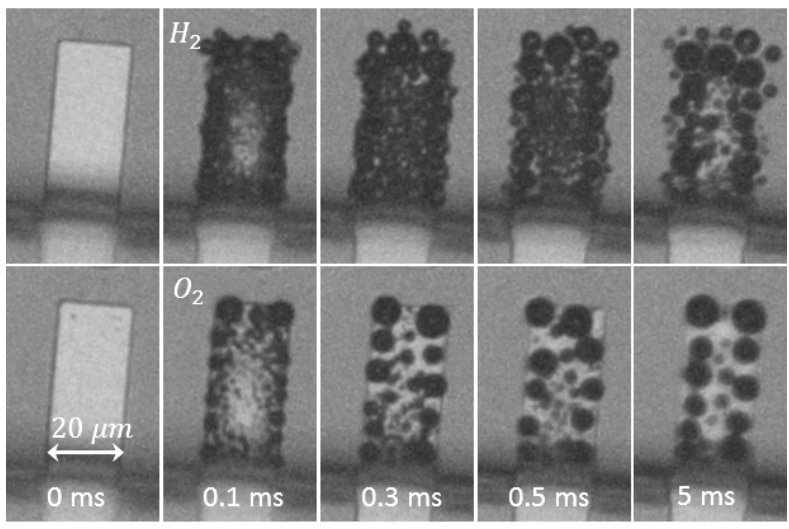

FIG. 5: Long-time dynamics of bubbles. Hydrogen (top row) or oxygen (bottom row) bubbles were produced by a voltage pulse of $100 \mu \mathrm{s}$ long. The images in each row were taken at different moments of time.

of gas appearing from nothing.

We consider Fig. 4 and explanations above as a strong support of the idea that in the short-time electrolysis we observe homogeneous formation of bubbles.

\section{Transition to long-time dynamics}

Let us consider now how transition to the usual longtime electrolysis happens. A negative or positive voltage pulse of $100 \mu$ s long was applied to the working electrode. The electrode was observed in different moments of time when the pulse was already switched off. Some results are shown in Fig. 5

It was already clear from Fig. 4 that nanobubbles growing from nucleus start to coalesce in some moment of time and form microbubbles (see bottom row in Fig. 41). Fast aggregation becomes possible due to high density of nanobubbles. Now from Fig. [5 one can see that significant changes happen between the images taken at 100 and $300 \mu \mathrm{s}$. The following dynamics becomes very slow. This is especially clear for the images at $0.5 \mathrm{~ms}$ and $5 \mathrm{~ms}$. It is expected that the long-time dynamics is controlled by the slow diffusion and slow coalescence.

The main conclusion that one can make from the short-time stroboscopic observations is that the nanobubbles are densely produced in the homogeneous nucleation regime. They exist for some time in the "invisible" form when they are small enough to scatter the light and are invisible for the stroboscope. At later moments they become visible but exist as separate nanobubbles. Later they start to aggregate to form microbubbles that are usually observed in the electrolysis. Extremely high current densities and relative supersaturations are observed.

\section{ALTERNATING SIGN PULSES}

Up to now we discussed only hydrogen and oxygen bubbles that are formed on different electrodes when negative or positive voltage is applied to the working electrode. However, there is a simple but effective procedure that allows formation of bubbles containing mixture of $\mathrm{H}_{2}$ and $\mathrm{O}_{2}$. Moreover, the gas composition can be controlled electrically. The idea is to use pulses of alternating polarity [21]. In this case hydrogen and oxygen will be produced locally above the same spot on the electrode. If the pulses are short enough to neglect the gas redistribution due to diffusion, then one can expect formation of bubbles containing both gases.

For homogeneous nucleation formation of a bubble containing both gases is favorable in comparison with two bubbles containing different gases. This is because even in the regime of homogeneous nucleation there is an energetic barrier to form the critical nuclei. For the second gas it is easier to diffuse inside of the existing bubble than to overcome the barrier and form a new nuclei.

\section{A. Reaction inside of nanobubbles}

While the frequency of alternating pulses is not very high the process proceeds similar to that for single polarity pulses. However, when frequency is higher than $f=20 \mathrm{kHz}$ (at room temperature) the visible gas suddenly disappears. This can be clearly seen in Fig. 6. The figure shows gas developed in the system after $1 \mathrm{~ms}$ of electrolysis. Negative potential pulses applied to the working electrode (bottom in each image) result in $\mathrm{H}_{2}$ formation above this electrode at different frequencies (right column). No significant dependence on the frequency is observed. Oxygen formed above the working electrode for positive pulses also does not show frequency dependence (middle column). The left column shows the situation for the alternating pulses applied to the working electrode. In this case we observe strong dependence on frequency. The alternating pulses repeated with the frequency $f=20 \mathrm{kHz}$ produce significant amount of gas. One can see that this gas is collected in bubbles which differ from that in the middle and right columns. Already at $f=50 \mathrm{kHz}$ only small volume of gas is observed. At $f=100 \mathrm{kHz}$ practically no gas is visible.

Where the gas has disappeared? The Faraday component of the current depends on frequency very weakly if depends at all. Because the pulses of single polarity produce the gas at all frequencies it is difficult to assume that the Faraday's law breaks at higher frequencies. Experimentally we established that the gas disappearance is related to the stoichiometric production of hydrogen and oxygen. There is no visible gas production while positive and negative halves of the pulse have the same duration. In this case two $\mathrm{H}_{2}$ molecules are produced for one $\mathrm{O}_{2}$ molecule. If we change the relative duration of the positive and negative parts of the pulse then the sto- 


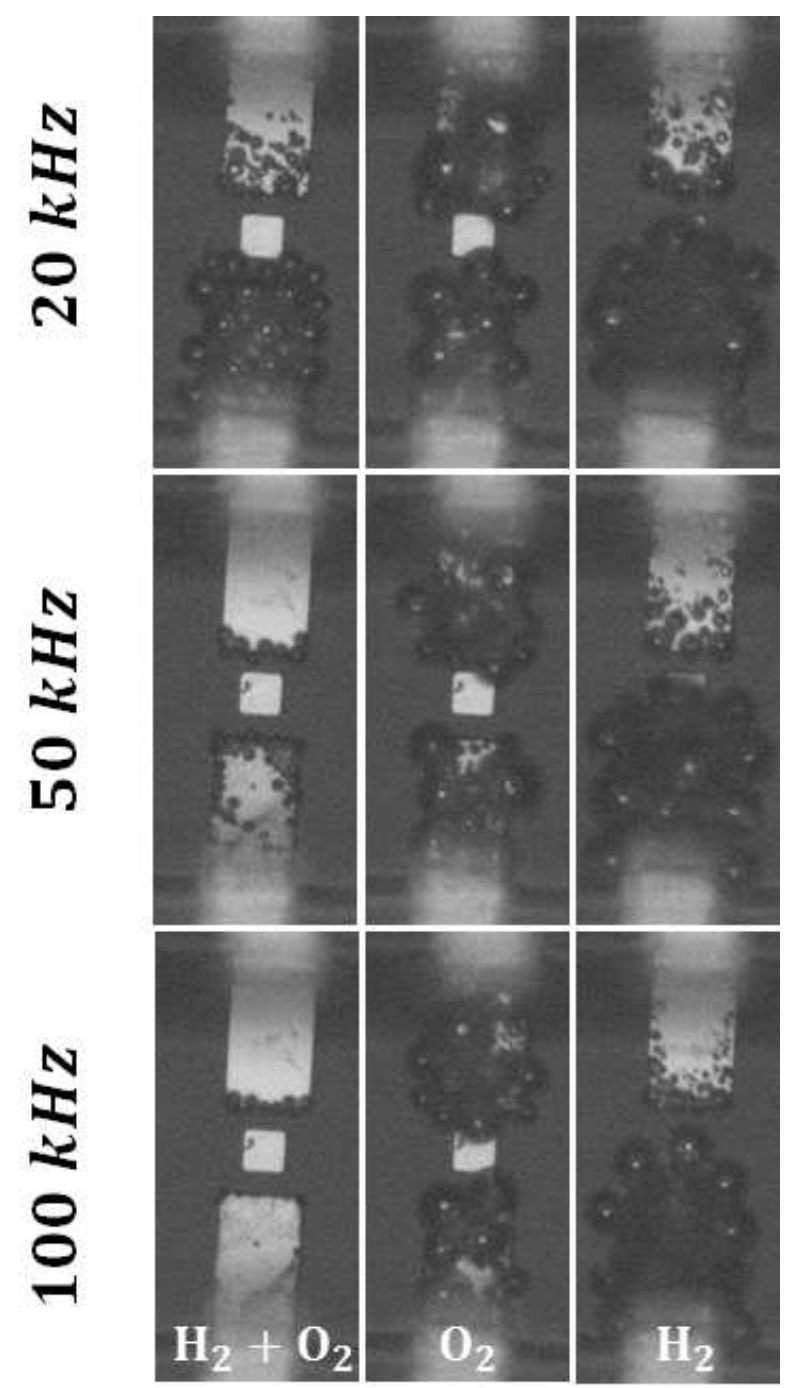

FIG. 6: Gas production with the negative pulses (right column), the positive pulses (middle column), and the alternating polarity pulses (left column) at different frequencies. For the alternating pulses the visible gas production disappears suddenly at higher frequencies.

ichiometric balance will be broken. In this case the gas reappeared in the system as shown in Fig. 7. In this figure $D$ is the duty cycle of the pulse that is the fraction of time when voltage on the working electrode is negative.

It seems reasonable to assume that the gas disappears due to a chemical reaction between gases happening in the solution. The reaction is hardly possible between separate dissolved gas molecules. For high supersaturation the molecules exist in the solution only a short time before entering nanobubbles as one can see in Fig. 3 and 4. It is possible that the reaction proceeds in nanobubbles containing stoichiometric mixture of $\mathrm{H}_{2}$ and $\mathrm{O}_{2}$. Of course, the bulk mixture of gases at room temperature and normal pressure will not react, but the gases confined in a small volume could behave differently. It is known that below a certain size, material properties can change drastically [39]. For example, the surface tension

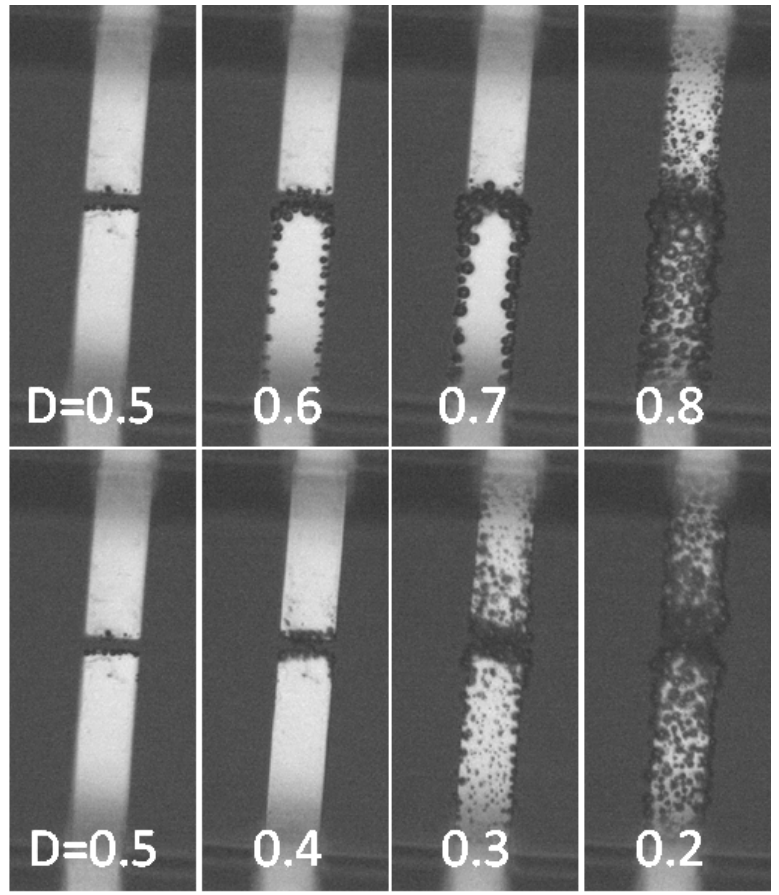

FIG. 7: Visible gas in the system after $200 \mu$ s of processing at $f=100 \mathrm{kHz}$ for different duty cycles $D$ of alternating polarity pulses. When the working electrode is equal time at positive and negative potential $(D=0.5)$ no gas is visible. If each pulse is more negative $(D>0.5)$, then excessive hydrogen is formed. For $D<0.5$ excessive oxygen appears. In all cases the deviation of $D$ from 0.5 results in reappearance of the visible gas.

can support metastable phases of nanocrystals [40] that exist only at high pressure for bulk materials. In liquids the surface tension results in significant pressure inside of nanobubbles. This pressure can shift the chemical equilibrium [41] and it is known also that fast dynamical processes play role for ignition of the reaction on the macroscale [42 44]. Nevertheless, the precise mechanism of the reaction is not clear at this moment.

We investigated also the effect of gas disappearance in different solutions such as $\mathrm{Na}_{2} \mathrm{SO}_{4}, \mathrm{NaCl}$, and $\mathrm{KI}$ dissolved in deionized water to similar concentrations $(\approx 1 \mathrm{M})$. The results of alternating pulse electrolysis in these solutions are shown Fig. 8, In the case of $\mathrm{NaCl}$ hydrogen and chlorine are produced. These gases can react with each other in the exothermic reaction with the enthalpy $\Delta H=-92 \mathrm{~kJ} / \mathrm{mol}$ considerably smaller than that for hydrogen and oxygen $\Delta H=-242 \mathrm{~kJ} / \mathrm{mol}$. One can see that the amount of visible gas decreases with the frequency increase but does not disappears completely. It means that the reaction starts spontaneously in smaller bubbles than for $\mathrm{Na}_{2} \mathrm{SO}_{4}$ solution. In the case of KI solution hydrogen and iodine are produced. The reaction between these molecules is endothermic, $\Delta H=25 \mathrm{~kJ} / \mathrm{mol}$, and cannot proceed spontaneously. Only weak dependence on $f$ is observed that is rather related to a small 


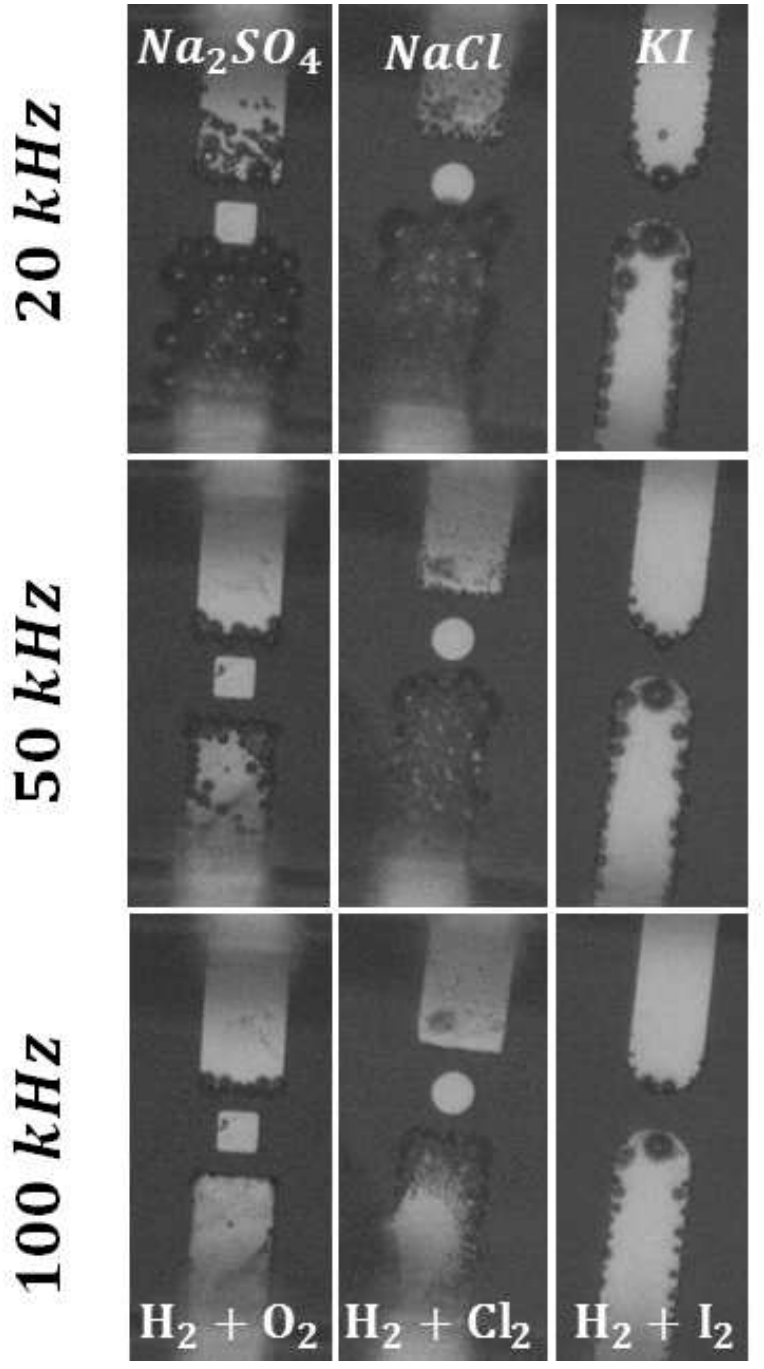

FIG. 8: Alternating pulse electrolysis in different solutions: $\mathrm{Na}_{2} \mathrm{SO}_{4}, \mathrm{NaCl}$, and $\mathrm{KI}$ dissolved in water. Each column corresponds to one of the solutions labeled on the top. Gases formed in the electrolysis are indicated in the bottom of the columns. Each row corresponds to the same frequency. The effect of gas disappearance clearly visible for $\mathrm{Na}_{2} \mathrm{SO}_{4}$ solution becomes less pronounced for $\mathrm{NaCl}$ and disappears at all for KI solution.

variation of the current.

Below we provide some additional experimental information that supports the hypothesis of chemical reaction inside of nanobubbles. However, the reaction is not the main topic of this paper; more information can be found in [21].

\section{B. Observations with vibrometer}

Observation of gas with a vibrometer is complimentary to observation with the stroboscope. The stroboscopic picture becomes visible when the bubbles significantly scatter the light. The nanobubbles with the size $d<\lambda / \pi$ are invisible for the stroboscope. On the other hand, strong scattering of the laser beam makes the interferometric (vibrometer) measurements impossible. Therefore, the vibrometer is effective for very small bubbles $d<\lambda / \pi$ where the stroboscope fails.

The vibrometer is sensitive to variations of the optical path $\Delta d(t)$ of the laser beam with time. The optical path is defined by the change of the refractive index $\Delta n$ due to the presence of gas in the solution. If we assume that within the laser beam the lateral distribution of gas is homogeneous then

$$
\Delta d=\int_{0}^{\infty} \mathrm{d} z\left[\Delta n_{1}(z)+\Delta n_{2}(z)\right]
$$

where $z$ is the vertical coordinate counting from the electrode surface, and $\Delta n_{i}(z)$ is the change of the refraction index due to gas $i$. Note that $\Delta d$ does not change if the gas molecules redistribute along $z$ but their number stays the same.

The refractive index of a liquid containing dissolved gas or gas collected in very small bubbles can be calculated using the Bruggman effective medium approximation [45]. If the volume fraction of gas is small, $f_{i} \ll 1$, then

$$
\Delta n_{i} \approx \frac{3 n_{0}\left(1-n_{0}^{2}\right)}{2\left(1+2 n_{0}^{2}\right)} f_{i},
$$

where $n_{0} \approx 1.34$ is the refractive index of the solution at $\lambda=633 \mathrm{~nm}$. The volume fraction of gas is expressed as $f_{i}=N_{i} / N_{\text {sol }}$, where $N_{i}$ is the concentration of $i$-th gas and $N_{\text {sol }} \approx 3.4 \times 10^{22} \mathrm{~cm}^{-3}$ is the concentration of the solution. If the gas in the volume cannot disappear, for example, due to chemical reaction, then the vibrometer signal must be proportional to the total gas flux produced by the Faraday current [21]:

$$
v(t)=-\frac{\partial \Delta d}{\partial t} \approx \frac{0.35}{N_{s o l}}\left[J_{1}(t)+J_{2}(t)\right],
$$

where $v(t)$ is the signal of the vibrometer and its opposite sign is defined by the instrument. The total flux of the produced molecules $J_{t o t}=J_{1}+J_{2}$ is a non-decreasing function of time.

We observed the process with an instrument Polytec MSA-400. The laser beam $(\lambda=633 \mathrm{~nm})$, with a diameter of $1.5 \mu \mathrm{m}$, was focused on the electrode in its center at small distance from the edge. The signal is presented in Fig. 9(a) and (b). When frequency of alternating sign pulses was low, large bubbles can be formed that scatter light significantly. In this case one cannot extract helpful information from the signal as shown in Fig. 9) (a) for $f=20 \mathrm{kHz}$. However, for higher frequencies the bubbles small in comparison with $\lambda / \pi$ are formed and one can clearly see the signal (see Fig $9(\mathrm{~b})$ ). The signal becomes weaker in the inner areas of the electrode in accordance with the current distribution. It disappears at all outside 

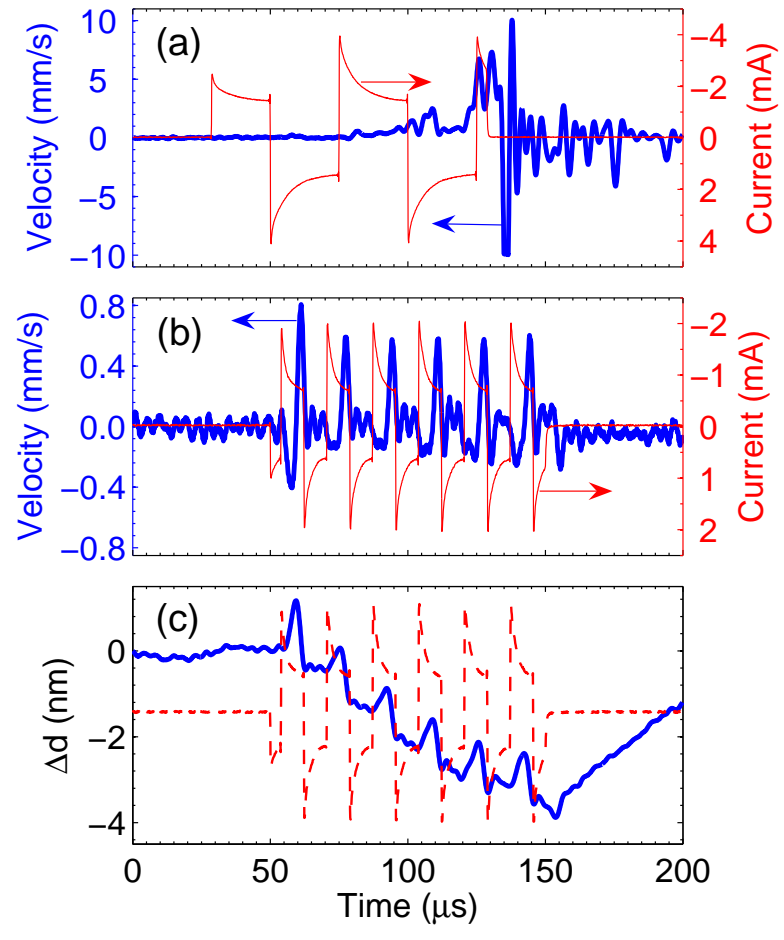

FIG. 9: Vibrometer signal. (a) Processing at low frequency, $f=20 \mathrm{kHz}$, results in formation of large bubbles strongly scattering the light. The thick blue curve is the signal and the thin red curve is the current. (b) The signal and current as functions of time for $f=60 \mathrm{kHz}$. The peaks in the signal are in phase with the voltage (current) pulses. (c) The signal in (b) integrated over time (thick blue). The red dashed line presents the current and is given here only for eye guidance.

of the electrodes. The latter demonstrates that the signal cannot be related to any kind of vibrations. Integration of the signal over time gives the variation of the optical path $\Delta d(t)$. The integrated signal is presented in Fig. 9(c).

The function $\Delta d(t)$ demonstrates prominent maximums that are in phase with the voltage pulses. These maximums mean periodic decrease of the gas concentration in the liquid. The only reasonable explanation for this decrease is the reaction between hydrogen and oxygen. Of course, the vibrometer signal is sensitive only to the overall concentration of gas in the liquid. It is not possible to say if the gas interacts as dissolved molecules or inside of nanobubbles. However, from observations of hydrogen and oxygen on the same time scale we know that each gas is collected in nanobubbles. It is hardly possible that the gases produced by alternating polarity pulses will behave differently.

The linear trend in $\Delta d(t)$ means that not all produced gas is burned in the reaction but its small part is left after each period. This residual gas exists mainly in the form of dissolved molecules. When the gas production is switched off the residual gas disappears. To all appearance it disappears also as the result of reaction because

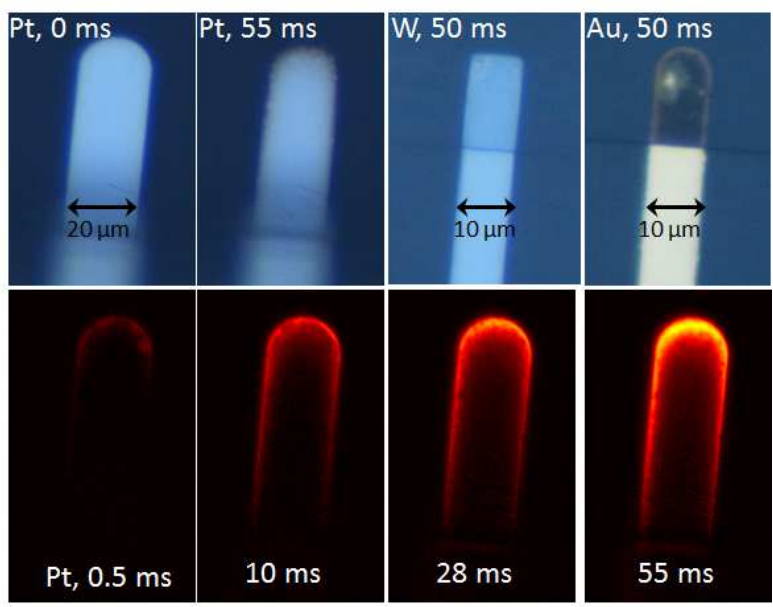

FIG. 10: Modification of the electrode surface. (top row) The left image shows untreated Pt electrode. The second image (from left to right) is the same electrode after $55 \mathrm{~ms}$ of processing. The third and forth images are for $\mathrm{W}$ and $\mathrm{Au}$ electrodes, respectively, after $50 \mathrm{~ms}$ of processing. (bottom row) Successive images of changes in the Pt electrode for different processing times. The colormap is changed for better visibility.

reduction of gas concentration due to diffusion has to proceed slower than observed. However, this point needs more attention.

\section{Modification of the surface of electrodes}

The effect of gas disappearance is accompanied by modification of the electrode surface (see Fig. 10). We call this phenomenon "wear" of electrodes. It never has been observed for single polarity electrolysis but well visible for the alternating polarity processing. The effect is observed for all investigated materials $\mathrm{Pt}, \mathrm{Pd}, \mathrm{W}$, and $\mathrm{Au}$ but manifests itself to a different degree. The strongest effect is observed for gold and the weakest one is for tungsten. It is in correlation with the material yield strength. The effect increases with the process time as shown in the bottom row of Fig. 10 .

The chemical origin of the surface modification can be excluded because it is observed for different metals. On the other hand, we can exclude the electrochemical origin of the modification because it is not observed for single polarity pulses. We expect that the surface modification happens as the result of local release of energy from the exploding nanobubbles that mechanically modify and shift the material of electrodes. This assumption naturally explains correlation with the material yield strength.

We observed only weak modification of the electrodes for the alternating pulse electrolysis in the $\mathrm{NaCl}$ solution. In the KI solution there was no visible modification of the electrodes at all. These facts are in good agreement 

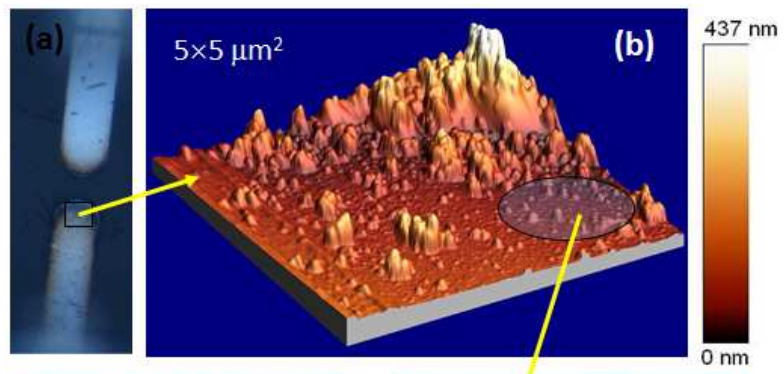

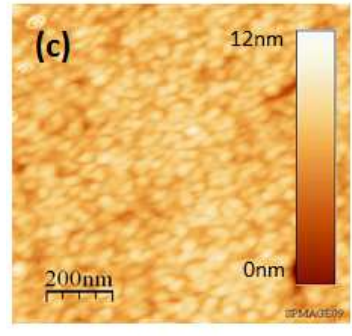

unexposed Pt

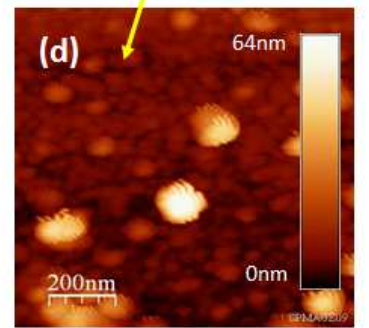

$55 \mathrm{~ms}$
FIG. 11: AFM scan of the modified surface. The process time was $55 \mathrm{~ms}$ (a) Optical image of the electrodes in air. An approximate location of the AFM scan is shown. (b) 3D topography of the electrode. Significant displacement of the material is observed. (c) Platinum surface before the process (normal roughness). (d) Modified roughness of the electrode after the process. The roughness changes on both lateral and vertical scales.

with the enthalpy of the reactions between $\mathrm{H}_{2}$ and $\mathrm{O}_{2}$, $\mathrm{Cl}_{2}$, or $\mathrm{I}_{2}$ : the larger the enthalpy the stronger is the modification of the surface.

The assumption of the mechanical modification is supported by the analysis of the modified surface with an atomic force microscope (AFM). The AFM scan of the modified electrode is shown in Fig. 11. One can see significant displacement of the material in Fig. 11(b), but the most important is that the process changes completely the roughness topography of the original platinum film as one can see in Fig. 11(c) and (d).

The experimental information collected so far on the alternating pulse electrolysis (see also [21]) provides strong evidence that the bubbles containing stoichiometric mixture of $\mathrm{H}_{2}$ and $\mathrm{O}_{2}$ gases explode as the result of spontaneous reaction between gases if the bubble size is smaller than $100-200 \mathrm{~nm}$. For pulses of low frequency the stoichiometric composition is reached for larger bubbles, which survive and can be observed with the stroboscope. Mechanism of the reaction in small bubbles is still not clear.

\section{DISCUSSION}

Let us summarize what we learned about the transient nanobubbles using different methods of observation. Of course, any bubble growing from a nuclei to its actual size gets through the phase when its size is in the nanorange. In this paper we have been concentrated on the bubbles that grow and live in the nanophase a very short time $\sim$ $10 \mu \mathrm{s}$. This regime is realized only when the bubbles are homogeneously produced nearby the electrode surface.

\section{A. Evolution of transient nanobubbles}

Homogeneous bubble production is closely related to the high current density that results in a huge supersaturation within the diffusion layer. The observed current density is large because we consider the system on the short-time scale $\tau \sim 10 \mu \mathrm{s}$. In this case the current is not restricted by the diffusion of electroactive species to the surface as it happens for the long-time electrolysis.

For single polarity pulses the critical nucleus are produced by the density fluctuations that is possible because of very high supersaturation. These nucleus start to grow diffusively. On the time scale $\tau \sim 10 \mu$ s they grow to nanobubbles of the size $d \lesssim 200 \mathrm{~nm}$ close to the limit of stroboscopic visibility. At longer time the separate nanobubbles start to aggregate to form microbubbles. Already at $\tau \sim 100 \mu \mathrm{s}$ most of the bubbles exist as microbubbles. Small increase of the temperature on $10-20$ degrees strongly intensify the bubble nucleation and grows as it should be for homogeneous bubble production. At higher temperature the microbubbles appeared already at $\tau \sim 30 \mu$ s or even earlier.

When all the gas is collected in microbubbles the supersaturation in the surrounding liquid is low and the process becomes very slow. The microbubbles slowly dissolve diffusively. For example, a bubble of $10 \mu \mathrm{m}$ in diameter will dissolve for a time of the order of $100 \mathrm{~ms}$.

The bubbles produced by the alternating polarity pulses evolve differently. The first half of the pulse produces high supersaturation of one gas, for example, $\mathrm{H}_{2}$. Nucleus of hydrogen are formed homogeneously and grown diffusively. The second half of the pulse produces $\mathrm{O}_{2}$ molecules in the same location above the electrode, but for oxygen it is energetically preferable to diffuse in the existing hydrogen bubble than to form a new $\mathrm{O}_{2}$ bubble. This is why the alternating polarity pulses produce bubbles containing mixture of hydrogen and oxygen. This mixture is stoichiometric if positive and negative halves of the pulse have the same duration.

If a hydrogen bubble has grown large enough before oxygen starts to diffuse inside, then such a bubble evolves similarly to the bubbles containing only one gas. The lower the frequency of the alternating pulses the longer time has a bubble to grow. The critical frequency is around $20 \mathrm{kHz}$. The critical size of the bubble cannot be estimated very precisely from the experiment, but roughly it is around $d \approx 150 \mathrm{~nm}$ [21]. For high frequency, $f>20 \mathrm{kHz}$, a bubble has no time to grow large. The gas mixture in the bubble becomes stoichiometric while its size is still under $150 \mathrm{~nm}$. It was established (see also 21]) that in such a bubble hydrogen and oxy- 


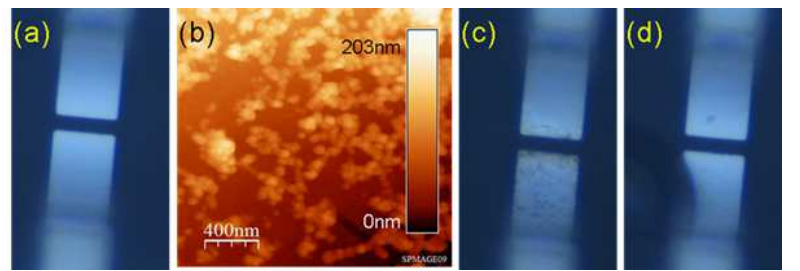

FIG. 12: (a) Clean electrodes. (b) AFM image of the electrode surface after fouling with $50 \mathrm{~nm}$ nanoparticles. (c) Optical image of the fouled electrodes. (d) After cleaning with homogeneously produced oxygen nanobubbles. Similar effect is observed for hydrogen nanobubbles.

gen react spontaneously with the bubble disintegration due to large released energy.

The mechanism of the reaction inside of nanobubbles is not clear. Bulk mixture of gases does not react at room temperature and the pressure that is equivalent to the Laplace pressure $P_{c}=4 \gamma / d \approx 30$ bar for $d=100 \mathrm{~nm}$. Of course, we cannot apply the classical combustion theory [46] to a strongly confined system such as nanobubble. To all appearance fast microsecond dynamics plays an important role for the combustion of mixture of gases. The detailed mechanism of the reaction is an important point that needs further attention.

The transient nanobubbles considered in this paper cannot help with the understanding of stability of the surface or bulk nanobubbles. The stabilization mechanism can work in these bubbles too but they cease to exists due to external reasons such as aggregation or combustion. The transient nanobubbles exist in a very thin diffusive layer above the surface, but we cannot say for sure if they attached to the surface or not. Relation between the transient and surface/bulk nanobubbles is an additional point that has to be understood.

\section{B. Possible applications}

We expect that the transient nanobubbles have a wide application potential because of their unique properties. For example, dense coverage with homogeneously produced nanobubbles can be used for surface cleaning in electronic industry and other applications. Figure 12 demonstrates the proof of concept, where the original clean electrodes were fouled with nanoparticles. The following homogeneous production of nanobubbles cleans the surface. The cleaning happens as the result of aggregation of nanobubbles that pulls the nanoparticles out of the surface. The cleaning effect was already stressed for the surface nanobubbles produced electrochemically [47] or with other methods [31, 48, 49]. However, homogeneous bubble production is better designed for local cleaning and has pronounced dragging effect on nanoparticles.

Especially interesting applications are expected for the exploding nanobubble phenomenon. Combustion inside of a nanobubble results in a significant energy deposition that is localized in a nanovolume. It can be applied, for example, to delete strongly adhered nanoparticles from masks or wafers in the electronic industry. One can easy change the bubble size by the electrical means (pulses frequency) varying significantly the energy of explosion.

Combustion in microscopic volumes is considered as difficult or impossible due to fast heat escape via the volume boundary 50]. The situation becomes different for nanovolumes. Exploding nanobubbles are the smallest combustion chamber realized in nature [51]. It opens up a principal possibility to fabricate an internal combustion engine microscopic in all three dimensions.

\section{CONCLUSIONS}

In this paper we described a special type of nanobubbles, which we call transient nanobubbles. These bubbles exist very short time $<100 \mu$ s before aggregating with other nanobubbles to form microbubbles or before exploding disintegration due to reaction between hydrogen and oxygen. The most distinctive feature for all transient nanobubbles is their homogeneous production in the electrochemical process. In contrast with the normal heterogeneous bubbling these bubbles nucleate due to fluctuations of liquid density.

Homogeneous nucleation is possible only for extremely high supersaturations that is related to very high current densities. These conditions can be met on a shot-time scale $\sim 10 \mu$ s when the diffusion of charged particles in the electrolyte does not restrict the current. On the short-time scale the gas produced on the electrode surface has no time to diffuse far away from this surface. For this reason high supersaturation can be realized only in a diffusion layer of thickness $\sim 100 \mathrm{~nm}$. In this thin layer only nanobubbles can be formed.

Short voltage pulses of fixed polarity repeated with some frequency $f$ can densely cover the electrode surface with nanobubbles containing hydrogen or oxygen. These nanobubbles aggregate and form microbubbles on the time scale $\sim 100 \mu \mathrm{s}$. The following dynamics of microbubbles becomes very slow.

Short voltage pulses of alternating polarity produce nanobubbles containing mixture of hydrogen and oxygen. If these bubbles are smaller than $150 \mathrm{~nm}$ the gases inside of them react spontaneously. As a result of this reaction significant energy is released in close proximity of the electrode surface. Precise mechanism of the spontaneous reaction inside of nanobubbles is still not clear and needs clarification.

\section{Acknowledgments}

We thank D. Lohse and J. Seddon for numerous discussions and K. Ma, H. Dijkstra, M. Siekman, and H. 
Wolferen for technical assistance. This work was partially supported by the Dutch Technological Foundation
(STW).
[1] S. Trasatti, J. Electroanal. Chem. 460, 1 (1999).

[2] R. de Levie, J. Electroanal. Chem. 476, 92 (1999).

[3] O. Khaselev, J. A. Turner, Science 280, 425 (1998).

[4] K. Zeng, D. Zhang, Prog. Energ. Combust. 36, 307 (2010).

[5] C. R. Neagu, J. G. E. Gardenier, M. Elwenspoek, J. J. Kelly, J. Micromech. Syst. 5, 2 (1996).

[6] C. G. Cameron, M. S. Freud, Proc. Natl. Acad. Sci. U.S.A. 99, 7827 (2002).

[7] S. Z. Hue, F. Sachs, D. X. Yang, H. D. Chopra, Anal. Chem. 74, 6392 (2002).

[8] D. E. Lee, S. A. Soper, W. Wang, Microsyst. Technol. 16, $381(2010)$.

[9] D. A. Ateya, A. A. Shah, S. Z. Hue, Rev. Sci. Instr. 75, 915 (2004).

[10] D. D. Meng, C.-J. Kim, Lab Chip 8, 958 (2008).

[11] S. Lee, E. Loth, C. Liu, Exp. Fluids 38, 672 (2005).

[12] J. Wang, M. Sullivan, S. Z. Hue, J. Microelectromech. Syst. 16, 1087 (2007).

[13] H. Matsushima, D. Kiuchi, Y. Fukunaka, K. Kuribayashi, Electrochem. Commun. 11, 1721 (2009).

[14] N. Shimizu, S. Hotta, T. Sekiya, O. Oda, J. Appl. Electrochem. 36, 419 (2006).

[15] N. L. Weinberg, K. Tomantscher, R. S. Feldstein, J. D. Gender, J. M. Rait, US patent 6638413 B1 (2003).

[16] N. P. Brandon, G. H. Kelsall, J. Appl. Electrochem. 15, 475 (1985).

[17] H. Vogt, R. J. Balzer, Electrochem. Acta 50, 2073 (2005).

[18] H. Vogt, J. Appl. Electrochem. 23, 1323 (1993).

[19] F. Hine, M. Yasuda, R. Nakamura, T. Noda, J. Electrochem. Soc. 122, 1185 (1975).

[20] H. Vogt, J. Appl. Electrochem. 13, 87 (1983).

[21] V. B. Svetovoy, R. G. P. Sanders, T. S. J. Lammerink, M. C. Elwenspoek, Phys. Rev. E 84, 035302(R) (2011).

[22] S. T. Lou, Z. Q. Ouyang, Y. Zhang, X. J. Li, J. Hu, M.Q. Li, F. J. Yang, J. Vac. Sci. Technol. B 18, 2573 (2000).

[23] R. Steitz, T. Gutberlet, T. Hauss, B. Klosgen, R. Krastev, S. Schemmel, A.C. Simonsen, G.H. Findenegg, Langmuir 19, 2409 (2003).

[24] M. Switkes, J. W. Ruberti, Appl. Phys. Lett. 84, 4759 (2004).

[25] Y. Wang, B. Bhushan, Soft Matter 6, 29 (2010).

[26] J. R. T. Seddon, D. Lohse, W. A. Ducker and V. S. J. Craig, Chem. Phys. Chem. 13, 2179 (2012).
[27] M. P. Brenner, D. Lohse, Phys. Rev. Lett. 101, 214505 (2008).

[28] S. Yang, S. M. Dammer, N. Bremond, H. J. W. Zandvliet, E. S. Kooij and D. Lohse, Langmuir 23, 7072 (2007).

[29] L. Zhang, Y. Zhang, X. Zhang, Z. Li, G. Shen, M. Ye, C. Fang and J. Hu, Langmuir 22, 8109 (2006).

[30] S. Yang, P. Tsai, E. S. Kooij, A. Prosperetti, H. J. W. Zandvliet and D. Lohse, Langmuir 25, 1466 (2009).

[31] S. Yang and Duisterwinkel, Langmuir 27, 11430 (2011).

[32] D. M. van den Broek, M. Elwenspoek, J. Micromech. Microeng. 18, 064003 (2008).

[33] A. J. Bard, L. R. Faulkner, Electrochemical methods, (Wiley, New York, USA 1980).

[34] C. F. Bohren and D. Huffman, Absorption and scattering of light by small particles, (Wiley, New York, USA 1983).

[35] P. G. Debenedetti, Metastable Liquids: Concept and Principles, (Princeton University Press, Princeton, NJ, 1996).

[36] L.D. Landau and E.M. Lifshitz Statistical Physics V.5 (Pergamon Press 1969).

[37] S.L. Girshick, C.-P. Chiu, J. Chem. Phys. 93, 1273 (1990).

[38] M. P. Anisimov, Russian Chem. Rev. 72, 591 (2003).

[39] G. Hodes, Adv. Mater. 19, 639 (2007).

[40] Q. S. Mei and K. Lu, Prog. Mater. Sci. 52, 1175 (2007).

[41] A. Sanfeld and A. Steinchen, Surf. Sci. 463, 157 (2000).

[42] A. V. Trotsyuk and P. A. Fomin, Combust. Expl., Shock Waves 28, 439 (1992).

[43] V. V. Golub et al., J. Loss Prevent Prosses Ind. 20, 439 (2007);

[44] F. L. Dryer et al., Combust. Sci. Tech. 179, 663 (2007).

[45] D. E. Aspnes, Thin Solid Films 89, 249 (1982).

[46] B. Lewis and G. von Elbe, Combustion, Flames and Explosions of Gases (Academic Press, New York, 1987).

[47] Z. Wu et al., J. Colloid Interface Sci. 328, 10 (2008).

[48] G. Liu, Z. Wu, and V. S. J. Craig, J. Phys. Chem. 112, 16748 (2008).

[49] G. Liu and V. S. J. Craig, Appl. Material Interface 1, 481 (2009).

[50] D. H. Lee, D. E. Pack, J. B. Yoon, S. Kwon, and E. Yoon, J. Micromech. Microeng. 12, 26 (2002).

[51] A. Smart, Physics Today 64, 17 (2011). 